\title{
Editorial
}

\section{Appraising investment risk}

\author{
Journal of Asset Management (2016) 17, 215-217. doi:10.1057/jam.2016.18
}

Appraising investment risk is not trivial, yet vital decisions depend on it.

In this special issue we want to express our determination to do as much as possible while fulfilling our mission of managing invested capital. We are determined to push the boundaries of science, opening up new investment solutions that are effective and fundamentally sound. To us, the key to a pertinent investment process lies in mastering the elementary aspects of the asset management process, not least the means that can be deployed for appraising investment risk.

In asset management, monitoring the risks inherent to the invested positions is a permanent concern, as is the need to be proactive, steering clear of risks that can be avoided. Risk Appraisal is inseparable from the investment risk itself.

It is not always evident how to discern investment risk in the first place. Investment risk is - according to the dictionary - the possibility of suffering a financial loss. How to assess such a possibility and how to measure the extent of the possible loss? There are no easy answers, and even if those elements are clearly present, perceiving them is no trivial matter.

Perception is - again from the dictionary the process of translating sensory impressions into a coherent view. It is a cognitive process with an initial measurement phase followed by an assessment phase, the latter further divided into acts of induction and of deduction. Given these concepts, how does one actually perceive investment risk? Let us examine a simple example where an investor holds a single asset.
The risk associated with holding the asset is in practice usually calculated by multiplying the amount invested $(x)$ by the asset return volatility $(\sigma)$, that is, $x \cdot \sigma$. As the asset return volatility is not observable, it must be estimated. Here is a deadlock in the perception process: one cannot make an estimate without having adopted preliminary assumptions. In other words, one cannot start the measurement phase before a prior induction phase. We are confined to measure by the norms we define, and remain oblivious to what we don't define.

The most commonly adopted norm is normality. Asset returns are assumed to follow a normal probability distribution function that is stationary, at least over the intended holding period of the asset. If we want to measure the volatility parameter of the distribution function, we need to assume that the function is stationary over a certain observation period. As we measure, the number of assumptions accumulate, weakening the perception process.

Another problem with the risk measure mentioned above lies in the perception of the outcome. The measure doesn't make a distinction between an unlikely severe loss or a more likely limited loss. The implicit assumption is that investors are indifferent towards those two possibilities, whereas in reality these events may be lived (perceived) very differently.

As a matter of fact, complementary risk measures are in use to capture unlikely severe losses, such as the Value At Risk measure or credit ratings. As the assumptions underlying these tail risk measures are different from the abovementioned 
mainstream risk measure - typically the normality assumption is dropped - it is difficult to conciliate the two. Judging, $e x-$ ante, whether a loss is severe or limited is a subjective matter and there is no standard in the investment profession to draw the line. Yet the choice of measure greatly affects how risk is perceived.

Let us look at a more realistic investment case where a multiple of $N$ assets are being held. The mainstream risk measure generalizes from $x \cdot \sigma$ to $x^{T} \cdot \Sigma \cdot x$, where $x$ is an $N$-vector containing the amounts invested and $\Sigma$ is the $\mathrm{N} x \mathrm{~N}$-covariance matrix between the asset returns. As $\Sigma$ contains many unknown parameters, assuming a normal and stationary probability distribution function no longer suffices. A more stringent risk model is needed in order to reduce the number of parameters to estimate, so as to make the parameters stable and reliable. Yet the more structure is imposed, the more the perception process is preconditioned and the more investors could hypothetically be misled.

Moreover, in the multivariate case a question of reference point arises: An asset, or a set of assets, is risky with respect to what? In technical terms, before risk estimation can start, the $N$ assets that constitute the investment universe and thus define the risk modelling setting must be decided. This choice once again predefines risk before the perception process has started. To take an example, when we assess a low-volatility investment strategy applied in equity space, the understanding of low volatility would be overthrown the moment fixed-income securities were introduced into the reference universe, showing that it is a matter of perception once again.

We believe that knowing the pitfalls and limits of the standing definitions of investment risk is elementary. It is an important focus of research for us, as the articles in this special issue illustrate.

In the first article written by Gianni Pola, the concept of entropy is introduced into the investment profession: the notion that the level of risk is somehow related to the level of information that is available. One can think of a ship at sea, for which visibility depends on the weather circumstances. In times of mist (lack of information), risk diversification becomes the priority or, as it were, a lighthouse in uncertain conditions.

In the second article, Ling-Ni Boon and Florian Ielpo argue that not only is the level of investment risk circumstantial, but the reward for taking on such risk is as well. The authors make evident that asset return is ultimately driven by growth prospects, in the developed world and nowadays increasingly in China, which are time-varying depending on the economic circumstances.

Their observation interconnects with Douglas Breeden's seminal Consumptionbased Capital Asset Pricing Model, which posits that parallel to the economic fundamentals, consumption drives the pricing process of assets. In the third article Douglas provides empirical evidence showing that consumption levels contain information that is relevant for equity and bond prices.

The discrepancy between reward and risk level is picked up again in the fourth article by Alessandro Russo, who gives empirical evidence of the much-commented low-risk anomaly in the context of equity investing. It remains an outstanding question whether stocks with a low price volatility truly outperform, or whether the risks related to such stocks are not appraised correctly.

Marielle De Jong and Lauren Stagnol raise the same question in the context of corporate bond investing, in the fifth article. Are the risks related to investing in bonds effectively reflected by the bond prices? As bond prices, distorted by a lack of market liquidity, may not convey information efficiently, price volatility or any measure involving prices may not lead to a sensible appraisal of risk. The authors establish a market-neutral bond 
positioning that is built on firm fundamentals, not prices.

In the sixth article, Marie Brière, Valérie Mignon, Kim Oosterlinck and Ariane Szafarz revisit the questions of appraising risk and reward in a timely investment setting. Central Banks are rethinking their position of the foreign reserves worldwide. Traditionally invested in US dollars, changes in the international monetary system and a renewed attention to return-to-risk efficiency push towards reassessing their asset allocation.
Pascal Blanqué Deputy Chief Executive Officer, Global Head of Institutional Business, Chief Investment Officer and member of the Executive Committee of Amundi Group.

Marielle De Jong Head of Fixed-income Quant Research at Amundi, Boulevard Pasteur, 75015 Paris. E-mail: marielle.dejong@amundi.com

Philippe Ithurbide Global Head of Research, Strategy and Analysis at Amundi.

Note from the Editor: I have been delighted to hand over editorial responsibilities to my editorial board colleague, Marielle de Jong for this special issue. She has shown excellent intellectual judgement and administrative acumen in putting together this issue and the selected material is a valuable addition to the academic and practitioner literature.

Stephen Satchell 